\title{
EVALUACIÓN DE UN SISTEMA DE TRATAMIENTO DOMÉSTICO PARA REÚSO DE AGUA RESIDUAL
}

\author{
Marco Antonio GARZÓN ZÚÑIGA ${ }^{1 *}$, Jazmín GONZÁLEZ ZURITA² y Raúl GARCÍA BARRIOS ${ }^{3}$
}

${ }^{1}$ Centro Interdisciplinario de Investigación para el Desarrollo Integral Regional, Unidad Durango, Instituto Politécnico Nacional. Sigma 119, Fraccionamiento 20 de noviembre II, Durango, Durango, México, C.P. 34220

${ }^{2}$ Universidad Nacional Autónoma de México, campus Instituto Mexicano de Tecnología del Agua, Paseo Cuauhnáhuac 8532, Progreso, Jiutepec, Morelos, México, C.P. 62550

${ }^{3}$ Centro Regional de Investigaciones Multidisciplinarias, Universidad Nacional Autónoma de México. Av. Universidad s/n, Circuito 2, Colonia Chamilpa, Cuernavaca, Morelos, México, C.P. 62210

*Autor para correspondencia: marco.cuerna@gmail.com

(Recibido junio 2015; aceptado noviembre 2015)

Palabras clave: sistema descentralizado, biofiltro orgánico, humedal construido, biopelículas, operación sencilla y económica

\section{RESUMEN}

En las zonas rurales y periurbanas de México sin drenaje, el agua residual (AR) es vertida al suelo, barrancas o arroyos, lo que genera problemas de salud y de contaminación. El objetivo del presente trabajo fue evaluar un sistema de tratamiento (ST) domiciliario, fácil y económico de operar y mantener, que consta de una fosa séptica (FS), un biofiltro (BF) y un humedal construido (HC) operados en serie. El BF utiliza como material de empaque filtrante, trozos de madera de elementos residuales de poda. $\mathrm{El} \mathrm{HC}$ es de flujo horizontal subsuperficial empacado con grava y sembrado con papiros y otras plantas ornamentales. El ST fue construido a escala real para una familia de dos a cuatro personas en zonas con baja disponibilidad y dotación per cápita de agua de $130 \mathrm{~L} / \mathrm{d}$ y un caudal de $400 \mathrm{~L} / \mathrm{d}$. Durante 220 días se estudió el efecto de aplicar dos diferentes caudales de operación (Q): $0.2 \mathrm{~m}^{3} / \mathrm{d}$ y $0.4 \mathrm{~m}^{3} / \mathrm{d}$, que influenciaron el tiempo de retención hidraúlico (TRH) de la FS $(5.5 \mathrm{~d}$ y $2.75 \mathrm{~d})$ y las velocidades de filtración (VF) del BF $(1.26 \mathrm{~m} / \mathrm{d}$ y $2.52 \mathrm{~m} / \mathrm{d})$ y del HC $(0.05 \mathrm{~m} / \mathrm{d}$ y $0.1 \mathrm{~m} / \mathrm{d})$. El sistema presentó buen desempeño en general. Al aplicar el caudal menor, la calidad del efluente respecto a la demanda bioquímica de oxígeno, coliformes fecales, huevos de helminto y grasas y aceites, cumplió con los límites máximos que establece la normativa mexicana (NOM-003-ECOL-1997) y estadounidense (USEPA 2004), que regulan el reúso de agua residual tratada en actividades tales como lavado de patios, riego de áreas verdes y uso en sanitarios. Para aplicar el caudal mayor se requiere desinfección adicional.

Key words: decentralized system, biofilter with organic bed, constructed wetland, biofilms, easy and economical operation

\begin{abstract}
In Mexico there are rural and urban marginal zones without sewage and the wastewater is directly discharged in the soil, revains and water courses, which generates health and
\end{abstract}


pollution issues. The objective of this work was to evaluate a system of easy and economical operation and maintenance that could treat the wastewater from one house. This wastewater treatment system (WTS) combines the operation in series of a septic tank (ST) a biofilter (BF) and a constructed wetland (CW). The BF uses wood chips as filtration media (FM). The CW works with a horizontal sub-superficial flow, and was packed with gravel and seeded with papyrus and other ornamental plants. The WTS was full scale and has a capacity for 2-4 people in regions with a low access and low per capita dotation of water $(130 \mathrm{~L} / \mathrm{d})$. The WTS was operated for 220 days to evaluate the effect of applying two different flows (Q): $0.2 \mathrm{~m}^{3} / \mathrm{d}$ and $0.4 \mathrm{~m}^{3} / \mathrm{d}$, affecting the hydraulic retention time (HRT) of the ST (5.5 d and $2.75 \mathrm{~d})$, the filtration velocity (FV) into the BF (1.26 $\mathrm{m} / \mathrm{d}$ and $2.52 \mathrm{~m} / \mathrm{d})$ and the FV into the CW $(0.05 \mathrm{~m} / \mathrm{d}$ and $0.1 \mathrm{~m} / \mathrm{d})$. The combined WTS presented an excellent performance, and when the slowest flow was applied, the effluent quality obtained was good enough to be directly reused in yards, washing activities, irrigation of green areas and toilet use, considering the analyzed parameters (biochemical oxygen demand, fecal coliforms, helmint eggs, oil and greases), and according to the Mexican norm NOM-003-ECOL-1997 and the American (USEPA 2004) regulations. If the second flow $\left(0.4 \mathrm{~m}^{3} / \mathrm{d}\right)$ wants to be applied, an additional disinfection is necessary.

\section{INTRODUCCIÓN}

En México solamente el $50.2 \%$ del agua residual (AR) generada y colectada en los sistemas municipales de alcantarillado es tratada (CONAGUA 2013) antes de ser vertida a los cuerpos receptores o reutilizada directamente en la agricultura (JiménezCisneros 2001), lo que representa un alto riesgo para la salud humana y para el ambiente. Esta situación se agrava en zonas urbanas periféricas y comunidades rurales sin drenaje, donde una opción sería tratar las AR en sistemas de tratamiento (ST) descentralizados que se instalan en el mismo lugar (in situ) que se generan y darles así un reúso. Lo anterior, por medio de tecnologías que requieran poca operación, bajo consumo energético y baja producción de lodos. Para poder reusar el agua tratada en actividades con contacto directo e indirecto como riego de áreas verdes y huertos, lavado de vehículos y patios, se debe cumplir con la normativa mexicana NOM-003-ECOL-1997 (SEMARNAT 1997) que establece los siguientes límites máximos permisibles de contaminantes: coliformes fecales (CF) $240 \mathrm{NMP} / 100 \mathrm{~mL}$, huevos de helminto $(\mathrm{HH}) 1 / \mathrm{L}$, grasas y aceites (G y A) $15 \mathrm{mg} / \mathrm{L}$, demanda bioquímica de oxígeno $\left(\mathrm{DBO}_{5}\right) 20 \mathrm{mg} / \mathrm{L}$ y sólidos suspendidos totales (SST) $20 \mathrm{mg} / \mathrm{L}$. Además, también debe cumplir con los límites que marca la NOM-001-SEMARNAT-1996 (SEMARNAT 1996) para metales pesados en embalses naturales y artificiales con uso en riego agrícola.

Actualmente, los ST naturales pueden cumplir con estos requisitos, sin embargo requieren de grandes superficies. Entre estos se encuentran las lagunas anaerobias de maduración y los humedales construidos
(HC). Otra tecnología que ha sido aplicada con éxito es la biofiltración sobre materiales orgánicos. La biofiltración es un proceso de filtración biológica lenta por percolación sobre materiales orgánicos (por ejemplo, residuos de poda) que actúan como un carbón activado que retiene contaminantes por adsorción, pero además, sobre este medio orgánico crece una biopelícula que permite la biodegradación de los contaminantes retenidos (Garzón-Zúñiga et al. 2012).

Por otra parte, los HC de flujo subsuperficial se construyen en canales empacados con grava como material de soporte para vegetación hidrófita emergente. En este tipo de HC el AR pasa a través del material de empaque (ME) y del entramado de raíces que se forma bajo el agua, el cual sirve de soporte para numerosos microorganismos que reciben el oxígeno directamente de las hidrófitas sin necesidad de aireación. Adicionalmente, las plantas absorben por su sistema radicular los minerales y nutrientes eutrofizantes. Ambos sistemas, BF (Vigueras-Cortés et al. 2013) y HC (Rivas et al. 2011), han demostrado ser eficientes para el tratamiento de AR domésticas. Sin embargo, presentan aspectos que pueden ser mejorados. Por ejemplo, el BF requiere de ME orgánico que debe de ser reemplazado cada 3 a 5 años aproximadamente. Si bien son materiales residuales, éstos se deben trabajar para elaborar un medio filtrante (MF) con características particulares. Una familia de cinco integrantes puede requerir aproximadamente 2 a $3 \mathrm{~m}^{3}$. Por su parte, si se utiliza el HC como sistema principal, requiere de un área aproximada de $25 \mathrm{~m}^{2}$, que difícilmente se encuentra disponible en una casa.

Por tal razón, el presente trabajo plantea combinar ambas tecnologías, proponiendo que el BF sea más 
pequeño. Su función sería dar un primer tratamiento para reducir una parte importante de la materia orgánica (MO), mientras que en el HC se busca remover los otros contaminantes tales como los nutrientes y los CF. Con la combinación de ambos procesos se pretende obtener un ST descentralizado eficiente y robusto, que ocupe menos espacio que un $\mathrm{HC}$ y que utilice menos MF orgánico que un BF típico, así como que produzca un efluente para reúso.

Por tal razón, los objetivos del presente trabajo fueron implementar, operar y evaluar el desempeño de un ST descentralizado de AR para casa habitación, que combina los procesos de fosa séptica, de biofiltración sobre lecho orgánico y de humedal construido.

\section{MATERIALES Y MÉTODOS}

\section{Sistema de tratamiento experimental|}

El sistema de tratamiento fosa séptica, biofiltro, humedal construido (FS+BF+HC) fue concebido para que en el biofiltro (BF) se degradara $50 \%$ de la MO (que queda después de la FS) y que en el HC se completara el tratamiento, al remover la MO, los nutrientes restantes y los CF. Por tal razón, el BF fue diseñado utilizando el $50 \%$ del área superficial recomendada en la bibliografía (Garzón-Zúñiga et al. 2008), siendo inicialmente la velocidad de filtración (VF) aplicada al BF el doble $(0.60 \mathrm{~m} / \mathrm{d})$ de la recomendada por los mismos autores, cuando el BF es el único proceso biológico de tratamiento.

El tren completo de tratamiento consta de una FS dividida en dos cámaras, donde se remueven las $\mathrm{G}$ y A y los sólidos suspendidos (SS). Posteriormente el AR es alimentada por pulsos ( 16 al día de $3 \mathrm{~min} \mathrm{c} / \mathrm{u}$ ) al BF por la parte superior a través de una red de distribución que permite repartir homogéneamente el AR sobre la superficie y ésta se infiltra sobre el MF orgánico y la biopelícula que crece sobre éste y sale por el fondo para pasar después al HC. Ahí se infiltra entre el ME, las raíces de las plantas y la biopelícula que crece sobre ellas para recibir un tratamiento de pulimento. Por último, el efluente llega a un tanque (enterrado) de almacenamiento para su reúso. El sistema fue construido como un piloto a escala real. La FS tiene un volumen útil de $1100 \mathrm{~L}$, el BF es cilíndrico con un diámetro de $0.45 \mathrm{~m}$ y una altura de $2 \mathrm{~m}$, por su parte el $\mathrm{HC}$ tiene forma de serpentín, ocupa un espacio de $7 \mathrm{~m}$ de longitud por $0.6 \mathrm{~m}$ de ancho y $0.6 \mathrm{~m}$ de profundidad (Fig. 1).

\section{Materiales de empaque}

El BF fue empacado con residuos de poda de ficus (Ficus benjamina) por ser un material muy abundante en diversas zonas del país y con un rápido crecimiento, cuyas características son una densidad de $97 \mathrm{~kg} / \mathrm{m}^{3}$, una porosidad en la cama de filtración del $88 \%$ y con un tamaño de partícula de $6.53 \mathrm{~cm}$ (largo) por $1.52 \mathrm{~cm}$ (ancho). Por su parte, el HC se empacó en 2 capas, la del fondo de $0.1 \mathrm{~m}$ de altura con grava de 1 pulgada y otra capa encima de $0.5 \mathrm{~m}$ de altura con grava de $1 / 2$ pulgada. Además, se emplearon 50 plantas a lo largo de los $7 \mathrm{~m}$ del HC: 30 papiros (Cyperus papyrus), cuatro cunas de Moisés (Spathiphyllum wallisii), cuatro alcatraces (Zantedechia aethiopica), cuatro lirios persa (Iris japonica), dos jengibres mariposa (Hedychium coronarium), dos hojas elegante (Alocasia sp.), cuatro platanillos (Heliconia sp.) y cuatro aves del paraíso (Strelitzia reginae).

\section{Arranque y acondicionamiento}

El sistema se arrancó alimentándolo con AR cruda que llega a la planta de tratamiento de aguas residuales (PTAR) del Instituto Mexicano de Tecnología del Agua. En el cuadro I se presenta la caracterización del AR. En esta etapa se aplicó un caudal bajo de $0.1 \mathrm{~m}^{3} / \mathrm{d}$ para acondicionar los procesos biológicos de tratamiento al hacer crecer una biopelícula que

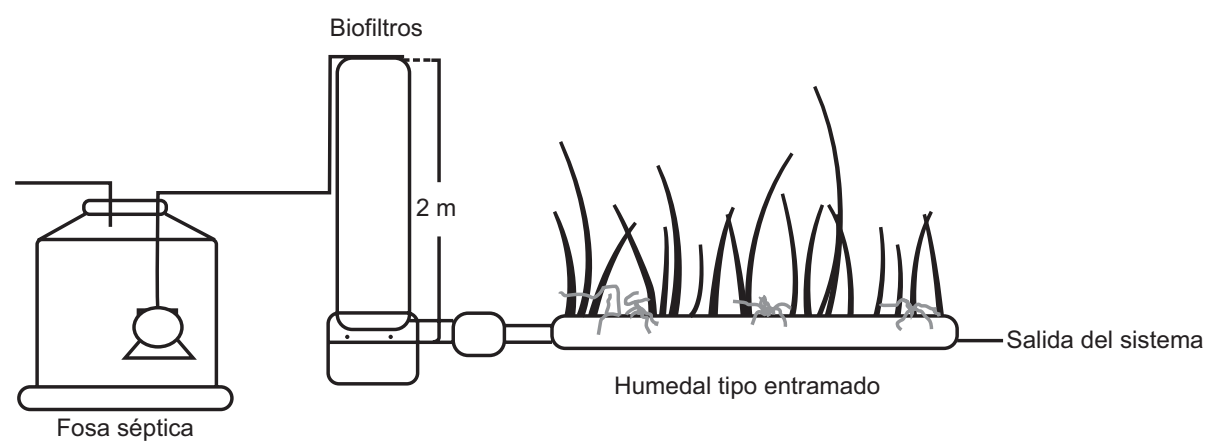

Fig. 1. Esquema del sistema de tratamiento decentralizado compuesto por fosa séptica, biofiltro y humedal construido 
CUADRO I. CARACTERIZACIÓN DE LAS AGUAS RESIDUALES CRUDAS UTILIZADAS

\begin{tabular}{lcc}
\hline Parámetro & $\begin{array}{c}\text { Valor promedio en agua } \\
\text { residual cruda } \pm \text { desviación estándar }\end{array}$ & $\begin{array}{c}\text { No. de } \\
\text { muestras }\end{array}$ \\
\hline pH (unidades) & $7.2 \pm 0.4$ & 24 \\
Nitrógeno total (mg/L) & $40.68 \pm 15.22$ & 6 \\
Nitrógeno amoniacal (mg/L) & $29.53 \pm 6.08$ & 25 \\
Fósforo total (mg/L) & $9.14 \pm 4.27$ & 6 \\
Fósforo de ortofosfatos (mg/L) & $7.72 \pm 1.87$ & 25 \\
Demanda química de oxígeno total (mg/L) & $401.6 \pm 129.9$ & 23 \\
Demanda bioquímica de oxígeno total $(\mathrm{mg} / \mathrm{L})$ & $142.8 \pm 25.9$ & 6 \\
Coliformes fecales $(\mathrm{MNP} / 100 \mathrm{~mL})$ & $7.57 \mathrm{E}+06 \pm 8.35 \mathrm{E}+06$ & 6 \\
Grasas y aceites $(\mathrm{mg} / \mathrm{L})$ & $36.2 \pm 25.8$ & 6 \\
Sólidos suspendidos totales $(\mathrm{mg} / \mathrm{L})$ & $139.4 \pm 62.1$ & 6 \\
\hline
\end{tabular}

degradara de forma estable $\mathrm{MO}$, así como para lavar el material orgánico del BF. A este caudal corresponde un tiempo de retención hidráulico (TRH) en la FS de $11 \mathrm{~d}$, una VF en el BF de $0.629 \mathrm{~m} / \mathrm{d}$ y una VF en el $\mathrm{HC}$ de $0.03 \mathrm{~m} / \mathrm{d}$. El sistema fue operado bajo estas condiciones hasta lograr una eficiencia de remoción sostenida del $\mathrm{BF} \geq$ al $50 \%$ de la demanda química de oxígeno total (DQOt), independientemente de lo que se remueva en la FS y una remoción total en todo el tren de tratamiento (FS $+\mathrm{BF}+\mathrm{HC}) \geq 80 \%$ de la DQOt del influente. Una vez alcanzada esta condición, se consideró que el ST estaba estabilizado.

\section{Efecto del caudal de operación}

Una vez estabilizado el ST, fue evaluado con dos caudales (Cuadro II). El primero $\left(\mathrm{Q}=0.2 \mathrm{~m}^{3} / \mathrm{d}\right.$ ) aumentó al doble la VF del arranque y el segundo $\left(0.4 \mathrm{~m}^{3} / \mathrm{d}\right)$ fue cuatro veces mayor. El cambio de caudal afectó directamente el TRH de la FS y del $\mathrm{HC}$, además de la VF del BF (Cuadro II).

\section{Muestreo y análisis}

Se realizó un muestreo puntual semanalmente (cada martes entre las 12:00 h y las 14:00 h) en los siguientes puntos: influente, salida de la FS, salida del BF y salida del HC. Cada muestra se analizó para $\mathrm{pH}$, nitrógeno amoniacal $\left(\mathrm{N}_{-} \mathrm{NH}_{4}{ }^{+}\right)$, fósforo de ortofosfatos $\left(\mathrm{P}^{-} \mathrm{PO}_{4}^{-3}\right)$ y DQOt. Adicionalmente, se realizó un único muestreo puntual y análisis de los principales parámetros de la normativa mexicana NOM-001-SEMARNAT-1996 (SEMARNAT 1996) para $\mathrm{CF}, \mathrm{DBO}_{5}$, G y A y $\mathrm{HH}$, para cada una de las condiciones de operación del sistema (arranque, caudal 1 y 2), una vez que este sistema se encontraba operando de forma estable en cada etapa. Los analisis se realizaron de acuerdo con la metodología que se presentan en el cuadro III.

\section{RESULTADOS Y DISCUSIÓN}

\section{Arranque y acondicionamiento}

Durante los primeros 50 días el AR cruda presentó una DQOt baja $(173 \pm 11 \mathrm{mg} / \mathrm{L})$ y el $51 \%$ fue removida en la FS, presentando a la salida una concentración promedio de $85 \pm 49 \mathrm{mg} / \mathrm{L}$. Esta eficiencia de remoción fue ligeramente superior a la que reporta la agencia de protección ambiental de Estados Unidos

CUADRO II. CONDICIONES EXPERIMENTALES DEL SISTEMA

\begin{tabular}{|c|c|c|c|c|c|c|c|}
\hline \multirow[b]{2}{*}{ Condición experimental } & \multirow{2}{*}{$\begin{array}{c}\mathrm{Q} \\
\left(\mathrm{m}^{3} / \mathrm{d}\right)\end{array}$} & \multicolumn{2}{|c|}{ TRH fosa séptica } & \multicolumn{2}{|c|}{ VF en BF } & \multicolumn{2}{|c|}{ TRH humedal } \\
\hline & & $\begin{array}{l}\text { Normal* } \\
\text { (días) }\end{array}$ & Pulso** & $\begin{array}{l}\text { Normal* } \\
\left(\mathrm{m}^{3} / \mathrm{m}^{2} \mathrm{~d}\right)\end{array}$ & Pulso** & $\begin{array}{l}\text { Normal* } \\
\text { (días) }\end{array}$ & Pulso** \\
\hline arranque & 0.1 & 11 & 0.36 & 0.629 & 18.8 & 12 & 0.4 \\
\hline 1 & 0.2 & 5.5 & 0.18 & 1.258 & 37.7 & 6 & 0.2 \\
\hline 2 & 0.4 & 2.75 & 0.09 & 2.515 & 75.4 & 3 & 0.1 \\
\hline
\end{tabular}

* Valor calculado de acuerdo con el caudal diario que se alimenta al sistema

** Valor calculado de acuerdo con el caudal instantáneo que se introduce en el sistema con cada pulso de alimentación

$\mathrm{TRH}=$ tiempo de retención hidráulico, $\mathrm{Q}=$ caudal, $\mathrm{VF}=$ velocidad de filtración, $\mathrm{BF}=$ biofiltro 
CUADRO III. MÉTODOS DE ANÁLISIS UTILIZADOS EN EL SEGUIMIENTO

\begin{tabular}{|c|c|}
\hline Parámetro & Método \\
\hline $\mathrm{pH}$ & Métodos estándar (APHA 1998) 4500 / USA EPA \\
\hline $\mathrm{N}-\mathrm{NH}_{4} / \mathrm{P}-\mathrm{PO}_{4}^{-3}$ & $\begin{array}{l}\text { Procedimiento Hach: } \mathrm{NH}_{4}{ }^{+} \text {-N Método } 8038 \text { (Hach) adaptado de los métodos estándar } \\
\text { (APHA 1998) 4500-NH3 (B) y (C) }\end{array}$ \\
\hline DQO total & Métodos estándar (APHA 1998) / Colorimétrico de reflujo cerrado. Método 5220 (C) \\
\hline Coliformes fecales & Métodos estándar (APHA 1998) / Tubos múltiples, Método 9230 (B). \\
\hline $\mathrm{HH}$ & Norma Oficial Mexicana NMX-AA-113-SCFI-2012 (SEMARNAT 2012). \\
\hline $\mathrm{DBO}_{5}$ total & Métodos estándar (APHA 1998) / Prueba de DBO al día 5, Método 5210 (B) \\
\hline G y A & Métodos estándar (APHA 1998) / Por Partición - Gravimetría, Método 5520 (B) \\
\hline
\end{tabular}

$\mathrm{DQO}=$ demanda química de oxígeno, $\mathrm{HH}=$ huevos de helminto, $\mathrm{DBO}_{5}=$ demanda bioquímica de oxígeno, $\mathrm{G}$ y $\mathrm{A}=$ grasas y aceites

de Norteamérica EPA-625-R-00-008 (USEPA 2002) para FS, operada a un TRH de 1 a 3 d, cuya eficiencia fue de $45 \%$ para la DQO. Esto se puede deber a que durante el arranque la FS operó con un TRH de 11 $\mathrm{d}$, mucho mayor al recomendado, debido a que se aplicó un caudal bajo $\left(0.1 \mathrm{~m}^{3} / \mathrm{d}\right)$ para hacer crecer una biopelícula en el BF y en el HC. El objetivo principal de una FS es retener sólidos por sedimentación y G y A por flotación. Por lo que se infiere que la eficiencia de remoción de la DQOt observada se debió a la retención de la DQO particulada o sedimentable. En este trabajo no se siguió la concentración de los SST, sin embargo, de acuerdo con la EPA-625-R-00-008 (USEPA 2002) la eficiencia de remoción de SST en una FS con un TRH de 1 a 3 d es de entre 50 y $70 \%$. Tomando en cuenta que el AR cruda utilizada tiene una concentración promedio de $139 \mathrm{mg} / \mathrm{L}$ (Cuadro I) se esperaría una concentración de salida de entre 70 y $42 \mathrm{mg} / \mathrm{L}$, misma que puede ser retenida en los procesos biológicos por filtración.

En la figura 2 se puede observar que la DQO de salida de la FS sigue los picos de DQO del AR de entrada. Esto se debe muy probablemente al tipo de muestreo, que al ser puntual midió una condición instantánea a diferencia de cuando se hace un muestreo compuesto en el cual se da tiempo para homogeneizar la muestra y reducir los picos.

Durante la primera semana de operación, la DQO de salida del BF fue $85 \%$ mayor a la de entrada (Fig. 2) y durante los primeros $50 \mathrm{~d}$, su eficiencia en la remoción de DQO fue sólo $16 \%$. Al respecto se ha reportado que la concentración de DQO en el efluente

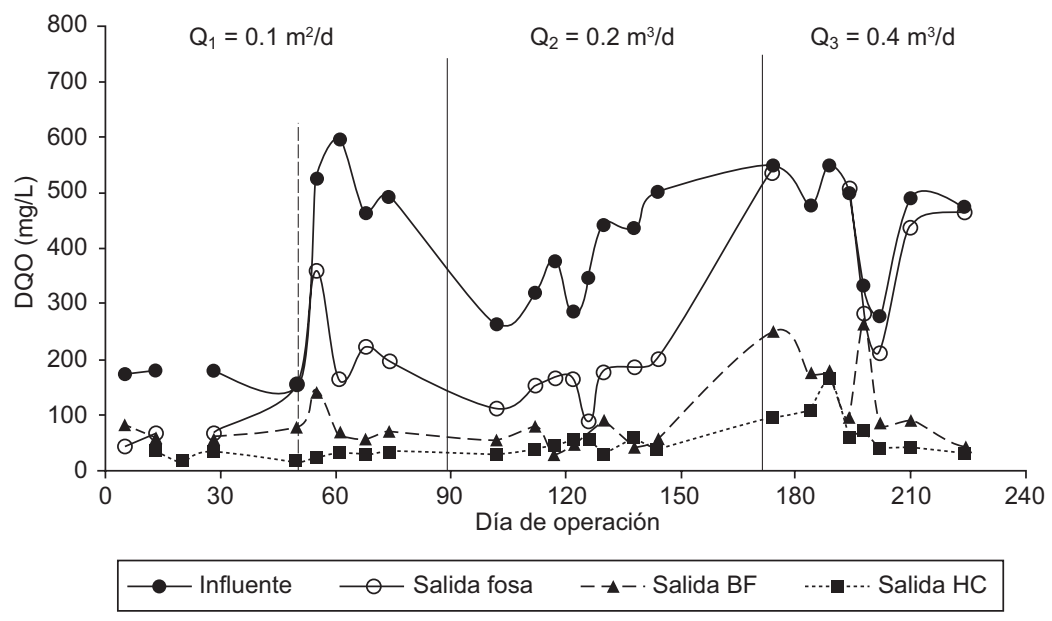

Fig. 2. Comportamiento de la demanda química de oxígeno (DQO) en cada proceso del sistema al aplicar diferentes caudales. $\mathrm{BF}=$ biofiltro, $\mathrm{HC}=$ humedal construido $\mathrm{Q}=$ caudal 
de diferentes biofiltros orgánicos aumentó durante el arranque, en porcentajes del 20 al $80 \%$ y hasta en $215 \%$ de forma temporal (Garzón-Zuñiga et al. 2008, Garzón-Zúñiga y Buelna 2011). Este comportamiento se atribuye a un lavado (solubilización) temporal de algunos componentes orgánicos de la madera.

A partir del día 55 de operación la DQO total del AR cruda aumentó en promedio a $520 \pm 57 \mathrm{mg} / \mathrm{L}$. Sin embargo, la FS mantuvo un desempeño similar, removiendo el $55 \%$ de la DQO total (seguramente DQO particulada) y presentó una concentración de salida de $236 \pm 87 \mathrm{mg} / \mathrm{L}$. En el BF la eficiencia de remoción de DQO aumentó de 16 a $64 \%$, siendo la concentración de salida de $84 \pm 39 \mathrm{mg} / \mathrm{L}$ (Fig. 2). Esto se debió, entre otros factores, a que se terminó el periodo de lavado del material orgánico y a que la biopelícula estaba más desarrollada, lo que permitió aumentar la remoción de la DQO soluble. Con esto se superó la eficiencia planteada inicialmente $(50 \%)$ como límite para considerar el sistema acondicionado. Este comportamiento se mantuvo durante $30 \mathrm{~d}$, con lo cual se consideró al ST listo para su evaluación. Durante ese tiempo el BF operó con una carga orgánica superficial (COS) de $0.148 \mathrm{~kg} \mathrm{DQO} / \mathrm{m}^{2} / \mathrm{d}$, similar a la utilizada por Vigueras et al. (2013) de $0.173 \mathrm{~kg} \mathrm{DQO} / \mathrm{m}^{2} / \mathrm{d}$ durante el arranque de un sistema de biofiltros empacados con fibra de agave.

Durante el periodo de acondicionamiento, el HC operó con una VF de $0.02 \mathrm{~m} / \mathrm{d}$ y se observó que los papiros crecieron rápidamente, proporcionando sombra y condiciones favorables para el crecimiento de las otras plantas de ornato. En cuanto a la DQO, en los primeros $50 \mathrm{~d}$, la eficiencia de remoción fue de $56 \%$. Durante la segunda etapa del arranque (del día 55 al 85) la COS aumentó a $0.0040 \mathrm{~kg} \mathrm{DQO} / \mathrm{m}^{2} / \mathrm{d}$, la eficiencia promedio incrementó a $64 \%$ y la eficiencia global de todo el sistema $(\mathrm{FS}+\mathrm{BF}+\mathrm{HC})$ fue de $94.17 \%$, con una concentración promedio en el efluente de $30 \pm 5 \mathrm{mg} / \mathrm{L}$
(Fig. 2). Esta VF está dentro del criterio recomendado por Wu et al. (2015) que es $<0.5 \mathrm{~m} / \mathrm{d}$ y además, es similar a la utilizada por Mietto et al. (2014) en un HC a escala real $(0.015 \mathrm{~m} / \mathrm{d})$ para remoción de nitrógeno.

\section{Efecto del caudal de operación del TRH y de la VF}

Durante la fase experimental 1, al aplicar un caudal de $0.2 \mathrm{~m}^{3} / \mathrm{d}$, la concentración promedio de DQO del AR fue $373 \mathrm{mg} / \mathrm{L}$ (Cuadro IV) y el TRH en la FS disminuyó a $5.5 \mathrm{~d}$, la VF al BF aumentó a $1.258 \mathrm{~m} / \mathrm{d}$ y la VF al HC se incrementó a $0.05 \mathrm{~m} / \mathrm{d}$ (Cuadro II). Bajo estas condiciones la FS continuó operando eficientemente y removió $58 \%$ de la DQO. En el BF la eficiencia de remoción de la DQO pasó de 64 a $62 \%$. Lo cual se puede explicar porque la COS que se aplicó al BF $\left(0.197 \mathrm{~kg} \mathrm{DQO} / \mathrm{m}^{2} / \mathrm{d}\right)$ fue sólo $32 \%$ mayor que la del arranque, a pesar de que la VF se duplicó debido a que la concentración de DQO en el influente disminuyó respecto a la etapa de arranque.

Por último, en el HC la COS aplicada $(0.0028 \mathrm{~kg}$ $\mathrm{DQO} / \mathrm{m}^{2} / \mathrm{d}$ ) fue $30 \%$ menor a la aplicada en la etapa de arranque a pesar de haber duplicado la VF a 0.05 $\mathrm{m} / \mathrm{d}$. Sin embargo, la eficiencia de remoción disminuyó ya que fue de tan sólo $25 \%$. Lo que a su vez afectó la eficiencia global, que se redujo de 94 a $87 \%$ con una concentración de salida de DQO de $44 \mathrm{mg} / \mathrm{L}$. Al respecto, Wu et al. (2015) mencionan que al aumentar la VF se observa una disminución en la remoción de contaminantes en los HC. Esta disminución podría estar relacionada con la disminución del TRH y con un arrastre de los contaminantes a la salida del HC.

Durante la fase experimental 2, al aumentar el caudal a $0.4 \mathrm{~m}^{3} / \mathrm{d}$, el TRH en la FS disminuyó a $2.7 \mathrm{~d}$, la VF en el BF aumentó a $2.515 \mathrm{~m} / \mathrm{d}$ y la VF al HC se incrementó a $0.10 \mathrm{~m} / \mathrm{d}$. Bajo estas condiciones la eficiencia en la FS se redujo a tan sólo $5 \%$ a pesar de que aparentemente el TRH estuvo dentro de los estándares recomendados por la EPA-625-R-00-008

CUADRO IV. CONCENTRACIÓN Y EFICIENCIAS DE REMOCIÓN DE LA DEMANDA QUÍMICA DE OXÍGENO (DQO)

\begin{tabular}{|c|c|c|c|c|c|c|c|c|}
\hline \multirow{2}{*}{$\begin{array}{l}\text { Caudal } \\
\left(\mathrm{m}^{3} / \mathrm{d}\right)\end{array}$} & \multicolumn{4}{|c|}{ DQO (mg/L) } & \multirow{2}{*}{$\begin{array}{c}\text { \% Remoción* } \\
\text { Fosa }\end{array}$} & \multirow{2}{*}{$\begin{array}{c}\% \text { Remoción* } \\
\text { BF }\end{array}$} & \multirow{2}{*}{$\begin{array}{c}\text { \% Remoción* } \\
\text { HC }\end{array}$} & \multirow{2}{*}{$\begin{array}{c}\text { \% Remoción** } \\
\text { Tren }\end{array}$} \\
\hline & $\begin{array}{c}\text { Influente } \pm \\
\text { D.S. }\end{array}$ & $\begin{array}{c}\text { Salida FS } \\
\pm \text { D.S. }\end{array}$ & $\begin{array}{c}\text { Salida BF } \\
\pm \text { D.S. }\end{array}$ & $\begin{array}{c}\text { Salida HC } \\
\pm \text { D.S. }\end{array}$ & & & & \\
\hline $0.1^{* * *}$ & $520 \pm 57$ & $236 \pm 87$ & $84 \pm 39$ & $28 \pm 7$ & $54.6 \%$ & $64.3 \%$ & $64.3 \%$ & $94.2 \pm 1.1 \%$ \\
\hline 0.2 & $373 \pm 83$ & $156 \pm 39$ & $59 \pm 20$ & $44 \pm 12$ & $58.0 \%$ & $62.2 \%$ & $25.3 \%$ & $87.7 \pm 4.3 \%$ \\
\hline 0.4 & $457 \pm 99$ & $434 \pm 122$ & $147 \pm 82$ & $77 \pm 44$ & $5.1 \%$ & $66.0 \%$ & $47.5 \%$ & $\pm 7.8 \%$ \\
\hline
\end{tabular}

*Eficiencia de remoción de la unidad de tratamiento (tecnología) en cuestión, tomando en cuenta la concentración de entrada y de salida a la unidad de tratamiento

** Eficiencia de remoción del tren de tratamiento que incluye todas las unidades de tratamiento y toma en cuenta la concentración a la entrada y salida del tren de tratamiento

***Segunda etapa del periodo de arranque (día 55 al 85 de operación)

$\mathrm{BF}=$ biofiltro, $\mathrm{HC}=$ humedal construido, $\mathrm{FS}$ = fosa séptica, D.S. = desviación estándar 
(USEPA 2002) que es de 1 a 3 d. Sin embargo, este comportamiento se puede explicar porque al alimentar el sistema por pulsos, el volumen de agua que ingresó en cada pulso generó un TRH durante los 3 min de la alimentación 30 veces mayor (Cuadro II), lo cual seguramente generó suficiente turbulencia para impedir que los sólidos sedimentaran. Por lo tanto, la DQO particulada salió junto con la DQO soluble de la FS durante el pico hidráulico de alimentación, provocando que la remoción de la DQOt fuera prácticamente inexistente. Esto podría haberse corroborado al realizar un análisis de SST, el cual desafortunadamente no fue realizado durante la investigación. Sin embargo, se puede observar que en el BF se obtuvo una remoción de $66 \%$ de la DQOt. Esto se puede explicar porque al ser este sistema un filtro, la mayor parte de la DQO particulada (SST) que salió de la FS pudo ser retenida por un proceso físico de filtración, con lo que se amortiguó el mal funcionamiento producido por los picos hidráulicos de la alimentación. Posteriormente, al pasar el AR por el HC se obtuvo una remoción de $47 \%$ adicional, con lo que se alcanzó una eficiencia global de $83 \%$ y un valor promedio de $77 \mathrm{mg} / \mathrm{L}$ de DQO en el efluente, eficiencia que está dentro de los estándares de remoción de un proceso de tratamiento secundario (Metcalf y Eddy 2003).

De forma global, la eficiencia de remoción en el BF se mantuvo cercana a $60 \%$ durante el arranque, acondicionamiento y las dos etapas experimentales, lo que demuestra la solidez de este proceso, mientras que la eficiencia en el HC disminuyó de $64 \%$ a $25 \%$ y a $47 \%$ al aumentar la VF.

La temperatura durante todo el periodo experimental osciló entre $17^{\circ} \mathrm{C}$ y $33^{\circ} \mathrm{C}$ y el pH se mantuvo entre 6.8 y 7.5. Estos valores resultaron adecuados para el desarrollo de la biopelícula y la acción de los procesos biológicos de depuración.

Respecto al N-NH${ }_{4}^{+}$, en la figura 3, se puede observar que la concentración de $\mathrm{N}-\mathrm{NH}_{4}{ }^{+}$en la FS casi no cambió, lo cual es normal por existir ausencia de oxígeno y por lo tanto de microorganismos nitrificantes. En el BF al inicio se observó una remoción de $60 \%$ del N-NH${ }_{4}^{+}$que fue disminuyendo hasta llegar a $13 \%$ y posteriormente la eficiencia volvió a aumentar y se mantuvo en valores entre $43 \%$ y $54 \%$. Este comportamiento en dos etapas se puede explicar si se considera que en la primera, los principales mecanismos de remoción son la absorción y la adsorción del $\mathrm{N}_{-} \mathrm{NH}_{4}{ }^{+}$, mientras que en la segunda son los procesos biológicos de nitrificación y asimilación. Al respecto, Buelna et al. (2011) mencionan que en los BF intervienen mecanismos de adsorción, absorción e intercambio iónico. Por otra parte Garzón-Zuñiga et al. (2005) estudiaron los mecanismos de remoción del nitrógeno en un BF con material orgánico y al realizar un balance de masa determinaron que: 1) durante los primeros $41 \mathrm{~d}$ la adsorción y absorción contribuyeron con el $33.6 \%$ de la remoción, del día 40 al 68 disminuyeron a $23 \%$ y posteriormente, a partir del día 70, ya no participaron en la remoción por la saturación del material; 2) Inicialmente, los procesos biológicos no contribuyeron de forma significante a la remoción de nitrógeno pero a partir del día $25,12 \%$ del nitrógeno fue nitrificado y a partir del día 68 más del $60 \%$ del N-NH${ }_{4}{ }^{+}$fue removido por nitrificación y desnitrificación simultáneas. En el presente estudio las eficiencias alcanzadas después del periodo de arranque (43 y $54 \%$ ) son menores a las reportadas en la bibliogarafia ya que el BF fue diseñado para

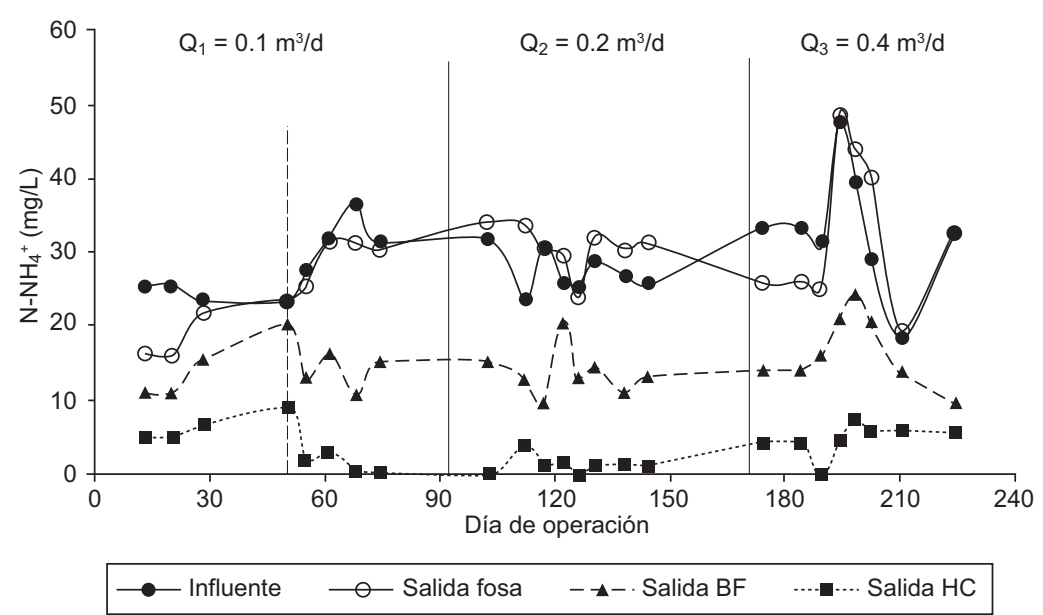

Fig. 3. Gráfica de $\mathrm{N}-\mathrm{NH}_{4}{ }^{+}$para $0.1,0.2$ y $0.4 \mathrm{~m}^{3} / \mathrm{d}$. $\mathrm{BF}=$ biofiltro, $\mathrm{HC}=$ humedal construido, $\mathrm{Q}=$ caudal 
CUADRO V. CONCENTRACIÓN Y EFICIENCIA DE REMOCIÓN DEL NITRÓGENO AMONIACAL $\left(\mathrm{N}-N H_{4}{ }^{+}\right)$

\begin{tabular}{ccccccccc}
\hline \multirow{4}{*}{$\begin{array}{c}\text { Caudal } \\
\left(\mathrm{m}^{3} / \mathrm{d}\right)\end{array}$} & $\begin{array}{c}\text { Influente } \\
\pm \text { D.S. }\end{array}$ & $\begin{array}{c}\text { Salida FS } \\
\pm \text { D.S. }\end{array}$ & $\begin{array}{c}\text { Salida BF } \\
\pm \text { D.S. }\end{array}$ & $\begin{array}{c}\text { Salida HC } \\
\pm \text { D.S. }\end{array}$ & $\begin{array}{c}\text { Femoción* } \\
\text { Fosa }\end{array}$ & $\begin{array}{c}\text { \% Remoción* } \\
\text { BF }\end{array}$ & $\begin{array}{c}\text { \% Remoción* } \\
\text { HC Remoción** } \\
\text { Tren }\end{array}$ \\
\hline 0.1 & $28.17 \pm 4.73$ & $24.6 \pm 6.3$ & $14.02 \pm 3.26$ & $\mathbf{3 . 7 7} \pm 3.3$ & $12.67 \%$ & $43.01 \%$ & $73.11 \%$ & $86.62 \%$ \\
0.2 & $28.02 \pm 3.06$ & $30.6 \pm 3.2$ & $13.92 \pm 3.32$ & $\mathbf{1 . 0 2} \pm 0.8$ & $-9.21 \%$ & $54.51 \%$ & $92.67 \%$ & $96.36 \%$ \\
0.4 & $33.1 \pm 8.3$ & $32.65 \pm 10.5$ & $16.625 \pm 4.8$ & $\mathbf{5 . 9 7} \pm 2.8$ & $1.36 \%$ & $49.10 \%$ & $64.08 \%$ & $81.96 \%$ \\
\hline
\end{tabular}

* Eficiencia de remoción de la unidad de tratamiento (tecnología) en cuestión, tomando en cuenta la concentración de entrada y de salida a la unidad de tratamiento

** Eficiencia de remoción del tren de tratamiento que incluye todas las unidades de tratamiento y toma en cuenta la concentración a la entrada y salida del tren de tratamiento

$\mathrm{BF}=$ biofiltro, $\mathrm{HC}=$ humedal construido, $\mathrm{FS}=$ fosa séptica, D.S. = desviación estándar

realizar sólo parte del tratamiento. La función del ME en los HC es más importante en la remoción del fósforo. Sin embargo, en el $\mathrm{HC}$, que también tiene aireación natural, ocurrieron procesos de nitrificación y asimilación. Además, se llevó a cabo una remoción adicional de nitrógeno de entre 20 y $40 \%$, con lo que se alcanzaron eficiencias globales entre $82 \% \mathrm{y}$ $96 \%$ de forma sostenida, independientemente de la VF aplicada en cada etapa, con concentraciones en el efluente entre 1 y $6 \mathrm{mg} / \mathrm{L}(\mathbf{C u a d r o} \mathbf{V})$.

La concentración de $\mathrm{P}_{-}-\mathrm{PO}_{4}^{-3}$ no disminuyó en la FS (Fig. 4) e incluso en varias ocasiones fue ligeramente superior (Cuadro VI). Esto se debe a que ocurre un proceso de hidrólisis del fósforo particulado lo cual incrementa los iones de fósforo. El aumento del $\mathrm{P}-\mathrm{PO}_{4}{ }^{-3}$ durante el periodo de arranque fue $<1 \%$ pero una vez que se acumularon lodos y se formó una biomasa en el fondo, el aumento de la concentración de $\mathrm{P}_{-} \mathrm{PO}_{4}{ }^{-3}$ estuvo entre 7 y $8 \%$ (Cuadro VI).
En el BF el P-PO ${ }^{-3}$ presentó un comportamiento diferente en cada etapa experimental. En el arranque la remoción inicial fue de $50 \%$ con tendencia a disminuir hasta desaparecer cerca del día 90. Posteriormente, a partir del día 140 en adelante, se observó una ligera remoción (11\%) de forma sostenida (Fig. 4). Este comportamiento en dos etapas, al igual que en el caso del N-NH${ }_{4}^{+}$, puede deberse primero a los procesos de retención por adsorción y asimilación para la formación de la biopelícula y, una vez saturado el MF, en la segunda etapa sólo a procesos de asimilación biológica. Lo anterior coincide con lo reportado por Garzón-Zuñiga y Buelna (2011) quienes probaron que en un biofiltro orgánico inicialmente se incrementa la concentración de fósforo debido al lavado de material orgánico y posteriormente se presenta una etapa de remoción relacionada con la capacidad de adsorción del ME.

En el HC se observó, de manera más evidente, una etapa inicial de remoción de $\mathrm{P}-\mathrm{PO}_{4}{ }^{-3}$ que tiende

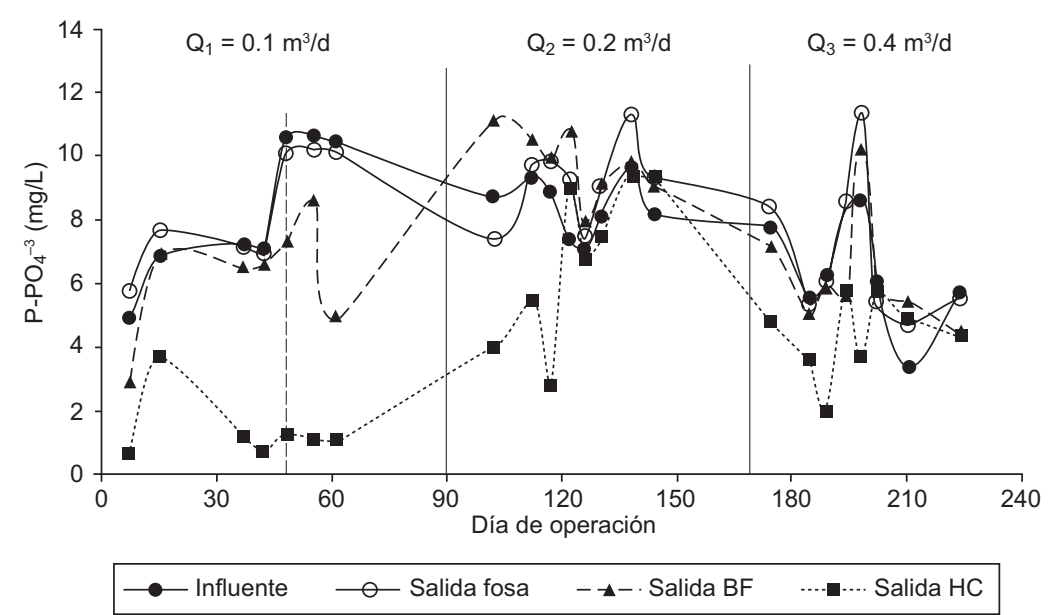

Fig. 4. Comportamiento del fósforo de ortofosfatos $\left(\mathrm{P}_{-} \mathrm{PO}_{4}{ }^{-3}\right)$ para $0.1,0.2$ y 0.4 $\mathrm{m}^{3} / \mathrm{d}$. $\mathrm{BF}=$ biofiltro, $\mathrm{HC}=$ humedal construido, $\mathrm{Q}=$ Caudal 
CUADRO VI. CONCENTRACIÓN Y EFICIENCIA DE REMOCIÓN DEL FÓSFORO DE ORTOFOSFATOS (P-PO4${ }^{-3}$ )

\begin{tabular}{cccccccrc}
\hline \multirow{2}{*}{$\begin{array}{c}\text { Caudal } \\
\left(\mathrm{m}^{3} / \mathrm{d}\right)\end{array}$} & $\begin{array}{c}\text { Influente } \\
\pm \text { D.S. }\end{array}$ & $\begin{array}{c}\text { Salida FS } \\
\pm \text { D.S. }\end{array}$ & $\begin{array}{c}\text { Salida BF } \\
\pm \text { D.S. }\end{array}$ & $\begin{array}{c}\text { Salida HC } \\
\pm \text { D.S. }\end{array}$ & $\begin{array}{c}\text { Pemoción* } \\
\text { Fosa }\end{array}$ & $\begin{array}{c}\text { \% Remoción* } \\
\text { BF }\end{array}$ & $\begin{array}{c}\text { Remoción* \% Remoción** } \\
\text { HC }\end{array}$ \\
\hline 0.1 & $8.28 \pm 2.28$ & $8.32 \pm 1.84$ & $6.27 \pm 1.84$ & $\mathbf{1 . 4 1} \pm 1.14$ & $-0.58 \%$ & $24.71 \%$ & $77.51 \%$ & $82.97 \%$ \\
0.2 & $8.46 \pm 0.92$ & $9.2 \pm 1.27$ & $9.83 \pm 1.05$ & $\mathbf{8 . 3 0} \pm 1.84$ & $-8.76 \%$ & $-6.86 \%$ & $15.60 \%$ & $1.91 \%$ \\
0.4 & $6.51 \pm 1.77$ & $6.99 \pm 2.28$ & $6.22 \pm 1.82$ & $\mathbf{5 . 2 5} \pm 1.57$ & $-7.29 \%$ & $11.06 \%$ & $15.57 \%$ & $19.42 \%$ \\
\hline
\end{tabular}

*Eficiencia de remoción de la unidad de tratamiento (tecnología) en cuestión, tomando en cuenta la concentración de entrada y de salida a la unidad de tratamiento

** Eficiencia de remoción del tren de tratamiento que incluye todas las unidades de tratamiento y toma en cuenta la concentración a la entrada y salida del tren de tratamiento

$\mathrm{BF}=$ biofiltro, $\mathrm{HC}=$ humedal construido, $\mathrm{FS}=$ fosa séptica, D.S. = desviación estándar

a decrecer (Fig. 4) y que estaría asociada a un fenómeno de adsorción en el ME, el cual se presentó durante el arranque y parte de la etapa experimental 1 hasta el día 130 de operación. Posteriormente y hasta el fin del periodo experimental, se observó una remoción de aproximadamente $15 \%$ (Cuadro VI) asociada a un equilibrio entre los procesos de adsorción, desadsorción y asimilación biológica por los organismos del HC (microorganismos y plantas). $\mathrm{Al}$ respecto, ha sido reportado que en los $\mathrm{HC}$, para obtener una remoción continua de fósforo es muy importante conocer la capacidad de adsorción del ME (Brix et al. 2001) y su potencial de saturación (Drizo et al. 2002), además de la capacidad de este ME para precipitar el fósforo (Arias y Brix 2005). Por esta razón, recientemente se han estudiado diferentes materiales: grava (Molle et al. 2005), apatita (Bellier et al. 2006), conchas de ostras (Seo et al. 2005) y cenizas y escoria de metal (Xu et al. 2006). Por otra parte, Korkuzus et al. (2005) reportan que en HC empacados con grava, como es el caso de este estudio, los principales mecanismos de remoción de fósforo son la adsorción y las reacciones de precipitación con $\mathrm{Ca}, \mathrm{Al}$, y Fe pero también la asimilación. Las eficiencias encontradas en el $\mathrm{HC}$ concuerdan con las reportadas en la bibliografía que varían entre $4 \%$ (He et al. 2007), 22\% (Akratos y Tsihrintzis 2007) y $67 \%$ cuando se utiliza grava fina (Korkusuz et al. 2005) en diferentes etapas de operación y saturación del ME.

Los CF fueron medidos al realizar un muestreo puntual, cuando el sistema se encontraba operando de forma estable, en cada etapa experimental (Cuadro VII) y se observó que: i) la concentración de $\mathrm{CF}$ del $\mathrm{AR}$ se mantuvo en valores entre $4.6 \mathrm{E}+06$ y $1.3 \mathrm{E}+08$, ii) en la FS se removió una unidad logarítmica, o no hubo remoción, iii) en el BF inicialmente sólo se removió una unidad logarítmica, pero con el tiempo la eficiencia aumentó a 3 unidades (etapas 2 y 3), lo cual coincide con lo reportado anteriormente por Garzón-Zúñiga et al. (2008). Estos autores proponen que la eficiencia de remoción de $\mathrm{CF}$, no es un proceso físico y químico únicamente, sino que una población de amebas tecadas, por ejemplo Euglypha rotunda, puede ejercer una presión de depredación sobre los $\mathrm{CF}$ al alimentarse de ellos. Estas amebas tecadas pueden degradar lignina y cellulosa, así como alimentarse de bacterias de vida libre (Alekperov y Snegovoya 2000, Gilbert et al. 2000, Nicolau et al. 2005) y han sido observadas viviendo en la biocenosis de biofiltros con empaques de astillas de diferentes maderas, de acuerdo con Garzón-Zúñiga et al. (2008). Estos autores proponen que la implantación y crecimiento de la población de dichas amebas estaría correlacionada con la disminución de los CF. iv) En el HC se removieron entre 2 y 3 unidades logarítmicas de $\mathrm{CF}$ con una concentración de salida inferior a $2.4 \mathrm{E}+02$ al aplicar los caudales de 0.1 y $0.2 \mathrm{~m}^{3} / \mathrm{d}(4.3 \mathrm{E}+01$ y $1.1 \mathrm{E}+02$, respectivamente), v) al aumentar el caudal la eficiencia disminuyó, para el caudal de $0.2 \mathrm{~m}^{3} / \mathrm{d}$ se alcanzó la concentración máxima permisible de la NOM-003-ECOL-1997 (ECOL 1997), no así para

CUADRO VII. CONCENTRACIÓN Y EFICIENCIA DE REMOCIÓN DE COLIFORMES FECALES (CF)

\begin{tabular}{cccccc}
\hline \multicolumn{4}{c}{$\begin{array}{c}\text { UFC Coliformes fecales } \\
\text { (NMP/100mL) }\end{array}$} & \multicolumn{2}{c}{$\begin{array}{c}\text { Remoción } \\
\text { Caudal } \\
\left(\mathrm{m}^{3} / \mathrm{d}\right)\end{array}$} \\
\cline { 2 - 5 } & $\begin{array}{c}\text { Influente } \\
\text { Salida } \\
\text { FS }\end{array}$ & $\begin{array}{c}\text { Salida } \\
\text { BF }\end{array}$ & $\begin{array}{c}\text { Salida } \\
\text { HC }\end{array}$ & \\
\hline 0.1 & $4.6 \times 10^{6}$ & $2.4 \times 10^{5}$ & $1.1 \times 10^{4}$ & 43 & $\mathbf{9 9 . 9 9 9}$ \\
0.2 & $1.7 \times 10^{7}$ & $1.3 \times 10^{8}$ & $9.2 \times 10^{5}$ & $1.1 \times 10^{2}$ & $\mathbf{9 9 . 9 9 9}$ \\
0.4 & $1.3 \times 10^{8}$ & $1.1 \times 10^{8}$ & $7.0 \times 10^{5}$ & $7.9 \times 10^{3}$ & 99.999 \\
\hline
\end{tabular}

$\mathrm{UFC}=$ unidades formadoras de colonias, $\mathrm{NMP}=$ Número mas probable, $\mathrm{BF}=$ biofiltro, $\mathrm{HC}=$ humedal construido, $\mathrm{FS}=$ fosa séptica 
el caudal de $0.4 \mathrm{~m}^{3} / \mathrm{d}$, aunque el valor estuvo muy cercano (7.9 E+03); en este caso se recomienda hacer una desinfección al final con cloro porque al ser baja la concentración de $\mathrm{MO}$ medida como $\mathrm{DBO}_{5}$ es muy bajo el riesgo de formar compuestos órganoclorados.

$\mathrm{El}$ análisis de detección de $\mathrm{HH}$ solamente fue realizado en el muestreo puntual que se hizo en cada etapa experimental cuando el sistema operaba de forma estable. En esos muestreos no se detectaron $\mathrm{HH}$ en el influente. Sin embargo, en la salida de la FS se registró un $\mathrm{HH}$ para los periodos experimentales 2 y 3 , pero a la salida del BF y del $\mathrm{HC}$ el número de $\mathrm{HH}$ fue cero. Estos resultados se pueden explicar porque se ha visto que la presencia de $\mathrm{HH}$ en el AR de entrada a una planta puede ser muy variable, a veces no trae $\mathrm{HH}$ y otras trae un número alto de ellos (Garzón-Zuñiga et al. 2006). Por otra parte, si bien en la FS los HH tienden a sedimentarse, al aplicar caudales altos que crean turbulencia los $\mathrm{HH}$ pueden resuspenderse y salir de la FS. Sin embargo, al entrar al BF y al $\mathrm{HC}$ estos son retenidos por filtración. La ausencia de $\mathrm{HH}$ a la salida del BF y del HC, concuerda con los resultados obtenidos por otros autores en BF con material orgánico (Garzón-Zúñiga et al. 2008, Vigueras-Cortés et al. 2013) y en un sistema similar de biofiltro con humedal de flujo vertical (Cervantes-Quiroz 2009), quienes reportan remoción del $100 \%$ de $\mathrm{HH}$.

$\mathrm{El}$ análisis de detección de $\mathrm{DBO}_{5}$ y de $\mathrm{G}$ y A también fue realizado sólo en el muestreo puntual en cada etapa experimental, cuando el sistema operaba de forma estable (Cuadro VIII). Respecto a la $\mathrm{DBO}_{5}$ en la FS, durante las etapas de arranque y primera experimental (TRH de $11 \mathrm{~d}$ y $5.5 \mathrm{~d}$ respectivamente), la remoción fue muy elevada (entre 52 y $72 \%$ ) lo que permite inferir que la FS funcionó adecuadamente al retener la mayor parte de la $\mathrm{MO}$ particulada y al dejar pasar la MO soluble. Pero en la segunda etapa experimental la eficiencia se redujo a $5 \%$ debido a que el TRH aplicado en cada pulso provocó picos hidráulicos (Cuadro I) con la consecuente resuspensión y salida de MO particulada de la FS. Sin embargo, en el BF la eficiencia de remoción fue más estable, entre 33 y $57 \%$, lo que compensó la baja eficiencia de la FS. Esto se explica porque el BF puede retener físicamente la MO particulada y además biodegradar la porción soluble. Cabe resaltar que cuando la FS presentó una eficiencia adecuada tanto el $\mathrm{BF}$ como el $\mathrm{HC}$ requirieron realizar una remoción menor para alcanzar las concentraciones que menciona la normatividad. Así, el porcentaje de remoción en el HC varió entre 22 y $96 \%$ al aplicar las tres VF. Sin embargo, la remoción global del sistema se mantuvo entre 86 y $99 \%$ y en el efluente se presentó una $\mathrm{DBO}_{5}$ menor o cercana a $20 \mathrm{mg} / \mathrm{L}$, que cumplen con la normativa mexicana de reúso de agua (NOM-003-ECOL-1997).

Respecto a las G y A (Cuadro VIII) se pudo observar que la FS presentó eficiencias de retención superiores al $80 \%$, a excepción del periodo en el que se aplicó el mayor caudal y se presentaron los picos hidráulicos por la alimentación por pulsos, lo que propició una salida de éstas con el efluente de la FS. Sin embargo, en todos los casos la concentración obtenida al final del tren de tratamiento respetó la normativa para descarga y reúso de AR tratada.

CUADRO VIII. CONCENTRACION Y EFICIENCIA DE REMOCIÓN DE LA DEMANDA BIOQUIIMICA DE OXÍGENO $\left(\mathrm{DBO}_{5}\right)$ Y DE LAS GRASAS Y ACEITES (G Y A)

\begin{tabular}{|c|c|c|c|c|c|c|c|c|}
\hline \multirow{2}{*}{$\begin{array}{l}\text { Caudal } \\
\left(\mathrm{m}^{3} / \text { día }\right)\end{array}$} & \multicolumn{4}{|c|}{$\mathrm{DBO}_{5}(\mathrm{mg} / \mathrm{L})$} & \multicolumn{3}{|c|}{ Remoción $\mathrm{DBO}_{5}(\%)^{*}$} & \multirow{2}{*}{$\begin{array}{l}\text { Remoción tren } \\
\text { completo }(\%) * *\end{array}$} \\
\hline & Influente & Salida FS & Salida BF & Salida HC & Salida FS & Salida BF & Salida HC & \\
\hline 0.1 & 146 & 70 & 33 & 1.2 & 52 & 53 & 96 & 99 \\
\hline 0.2 & 175 & 48 & 31 & 24 & 72 & 35 & 22 & 86 \\
\hline \multirow[t]{2}{*}{0.4} & 138 & 131 & 57 & 16 & 5 & 57 & 71 & 88 \\
\hline & \multicolumn{4}{|c|}{ G y A $(m g / L)$} & \multicolumn{3}{|c|}{ Remoción G y A (\%)* } & \\
\hline 0.1 & 43.4 & 7.5 & 4.9 & 4.9 & 83 & 34 & 0 & 89 \\
\hline 0.2 & 57.7 & 8.2 & 4.8 & 4.8 & 86 & 41 & 0 & 92 \\
\hline 0.4 & 7.6 & 6.3 & 8.2 & 7.3 & 17 & -30 & 22 & 16 \\
\hline
\end{tabular}

*Eficiencia de remoción de la unidad de tratamiento (tecnología) en cuestión, tomando en cuenta la concentración de entrada y de salida a la unidad de tratamiento

** Eficiencia de remoción del tren de tratamiento que incluye todas las unidades de tratamiento y toma en cuenta la concentración a la entrada y salida del tren de tratamiento

$\mathrm{BF}=$ biofiltro, $\mathrm{HC}=$ humedal construido, $\mathrm{FS}=$ fosa séptica 
Las eficiencias de remoción obtenidas al aplicar al $\mathrm{BF}$ una VF de $2.5 \mathrm{~m} / \mathrm{d}$ y al $\mathrm{HC}$ de $0.952 \mathrm{~m} / \mathrm{d}(83 \%$ de la DQO, $88 \%$ de la $\mathrm{DBO}_{5}, 82 \%$ del $\mathrm{N}_{-} \mathrm{NH}_{4}{ }^{+}, 15 \%$ del $\mathrm{P}_{-} \mathrm{PO}_{4}{ }^{-}$y 5 unidades log de CF), son similares a las reportadas por Cervantes-Quiroz (2009), quien trabajó un BF empacado con tabachín acoplado a un $\mathrm{HC}$ de flujo vertical subsuperficial empacado con tezontle y plantas de la especie Spathiphyllum wallisii para tratar AR sanitaria a una VF en el BF de $2.9 \mathrm{~m} / \mathrm{d}$ y de $0.245 \mathrm{~m} / \mathrm{d}$ en el HC. Este autor obtuvo las siguientes remociones de contaminantes: $98 \%$ de la DQO, $95 \%$ del $\mathrm{N}-\mathrm{NH}_{4}{ }^{+}$y $73 \%$ del P-PO ${ }_{4}^{-3}, 3$ a 5 unidades log de $\mathrm{CF}$ y $100 \%$ de $\mathrm{HH}$. La diferencia en cuanto a la eficiencia de remoción de MO puede explicarse porque en el trabajo mencionado, los investigadores enriquecieron el AR con glucosa y fosfato de amonio aminoácido, es decir MO y nutrientes en forma soluble mientras que en este estudio, al utilizar un efluente real, buena parte de la $\mathrm{MO}$ está en forma particulada, que es más difícil de biodegradar. Mientras que la diferencia en la remoción de aniones puede estar relacionada con el tipo de empaque del $\mathrm{HC}$, ya que el tezontle tiene una mayor capacidad de adsorción que la grava.

En relación con las especies de plantas, se observó que los papiros fueron los que mejor se adaptaron a las condiciones del HC. Estos organismos presentaron raíces profundas, alta capacidad de propagación y resistencia. Las plantas ornamentales crecieron bien pero el lirio persa presentó poca adaptabilidad.

\section{CONCLUSIONES}

El sistema combinado de fosa séptica (FS), biofiltración sobre lecho orgánico y humedal construido (HC) de flujo horizontal subsuperficial, demostró ser robusto ya que presentó buenas eficiencias de remoción de contaminantes. Cuando alguno de los procesos que lo componen obtuvo un mal desempeño, el buen desempeño de los otros dos procesos permitió mantener una buena eficiencia global.

Los resultados demostraron que el sistema presentó un buen desempeño al aplicar los dos caudales $\left(0.2 \mathrm{~m}^{3} / \mathrm{d}\right.$ y $\left.0.4 \mathrm{~m}^{3} / \mathrm{d}\right)$, pero la calidad del efluente que se obtuvo al aplicar el menor (DQO $\leq$ $44 \pm 12 \mathrm{mg} / \mathrm{L} ; \mathrm{DBO}_{5} \leq 24 \mathrm{mg} / \mathrm{L}, \mathrm{P}_{-} \mathrm{PO}_{4}{ }^{-3}<5 \mathrm{mg} / \mathrm{L}$, $\mathrm{N}-\mathrm{NH}_{4}^{+} \cdot \leq 3.77 \pm 3.3 \mathrm{mg} / \mathrm{L}, \mathrm{G}$ y A $\leq 4.8 \mathrm{mg} / \mathrm{L}, \mathrm{HH}$ $=0 \mathrm{y} \mathrm{CF}=1.1 \mathrm{E}+02 \mathrm{NMP} / 100 \mathrm{~mL}$ ) cumplió para los parámetros evaluados con la norma mexicana (NOM-003-ECOL-1997). Esta norma, contempla el reúso urbano de agua con contacto directo considernado los siguientes límites máximos:
$\mathrm{CF}=2.4 \mathrm{E}+02 \mathrm{NMP} / 100 \mathrm{ml}, \mathrm{HH}=1, \mathrm{G}$ y $\mathrm{A}=15$ $\mathrm{mg} / \mathrm{L}$ y $\mathrm{DBO}_{5}=20 \mathrm{mg} / \mathrm{L}$. Respecto a la normativa estadounidense (USEPA 2004), en ella se establece como límites máximos de descarga los siguientes valores: $\mathrm{CF}=2.3$ a $7.5 \mathrm{E}+01 \mathrm{NMP} / 100 \mathrm{ml}, \mathrm{SST}=5$ a $30 \mathrm{mg} / \mathrm{Ly} \mathrm{DBO}_{5}=5$ a $30 \mathrm{mg} / \mathrm{L}$ por lo que puede reutilizarse directamente en actividades tales como lavado de patios, riego de áreas verdes y uso en sanitarios. Mientras que en el caso del caudal mayor es necesario aplicar una desinfección al final del proceso para cumplir con los límites de CF. Pero se debe verificar que el efluente cumpla también con SST y metales pesados de acuerdo con lo estipulado en la NOM-001-SEMARNAT-1996.

El sistema puede considerarse como una alternativa viable y recomendable para el tratamiento y reúso in situ de AR en comunidades rurales, urbanas marginadas, conjuntos habitacionales y/o unidades unifamiliares sin acceso a la red de drenaje.

\section{REFERENCIAS}

Akratos C.S. y Tsihrintzis V.A. (2007). Effect of temperature, HRT, vegetation and porous media on removal efficiency of pilot-scale horizontal subsurface flow constructed wetlands. Ecol. Eng. 29, 173-191. DOI: 10.1016/j.ecoleng.2006.06.013.

Alekperov I. y Snegovaya N. (2000). The fauna of testate amoebae (Rhizopoda, Testacea) in freshwater basins of Apsheron peninsula. Protistology 1, 135-147.

APHA (1998). Standard Methods for the Examination of Water and Wastewater. 20a ed. American Public Health Association. Washington, EUA, 1325 pp.

Arias C.A y Brix H. (2005). Phosphorus removal in constructed wetlands: can suitable alternative media be identified?. Wat. Sci. Tech. 51, 267-273.

Bellier N., Chazarenc F. y Comeau Y. (2006). Phosphorus removal from wastewater by mineral apatite. Water Res. 40, 2965-2971. http://dx.doi.org/10.1016/j.watres.2006.05.016.

Brix H, Arias C.A. y del Bubba M. (2001). Media selection for sustainable phosphorus removal in subsurface flow constructed wetlands. Wat. Sci. Tech. 44, 47-54.

Buelna G. y Bélanger G. (1990). Biofiltration a base de tourbe pour le traitement des eaux usées des petites municipalités. Sciences et Techniques de 1'eau 23, 259-264.

Buelna G., Garzón-Zuñiga M.A. y Moeller G. (2011). Los biofiltros de empaque orgánicos: una alternativa simple, robusta y eficiente para el tratamiento de aguas residuales en zonas rurales. Ide@s CONCYTEG 6, 541-552. 
Cervantes-Quiroz A.L. (2009). Sistema combinado para el tratamiento de aguas residuales para viviendas unifamiliares. Tesis de Maestría en Ingeniería Ambiental. Facultad de Ingeniería. Universidad Nacional Autónoma de México. Ciudad de México, México, 98 pp.

CONAGUA (2013). Inventario Nacional de Plantas Municipales de Potabilización y de Tratamiento de Aguas Residuales en Operación. Comisión Nacional del Agua. Subdirección General de Agua Potable, Drenaje y Saneamiento. Gerencia de Potabilización y Tratamiento de Aguas Residuales. Inventario. Ciudad de México, México, 298 pp.

Dirzo A., Comeau Y., Forget C. y Chapuis R. (2002). Phosphorus saturation potential: A parameter for estimating the longevity of constructed wetland system. Environ. Sci. Technol. 36, 4642-4648. DOI: 10.1021/es011502v.

Dubé R. (1996). Traitement du lisier de porc par biofiltration sur milieu organique. Tesis de Maestría. Facultad de Ingeniería Civil. Universidad Laval. Ste. Foy Québec, Canadá, 68 pp.

ECOL (1997). Norma Oficial Mexicana NOM-003ECOL-1997. Que establece los límites máximos permisibles de contaminantes para las aguas residuales tratadas que se reúsen en servicios al público. Secretaría de Medio Ambiente y Recursos Naturales. Diario Oficial de la Federación. 21 de septiembre de 1998.

Jiménez Cisneros B. L. (2001). La contaminación ambiental en México, causas, efectos y tecnología apropiada. Limusa, Ciudad de México, México, 138 pp.

Garzón-Zúñiga M.A. y Buelna G. (2011). Treatment of wastewater from a school in a decentralized filtration system by percolation over organic packing media. Wat. Sci. Tech. 64, 1169-1177.

Garzón-Zúñiga M. A., Buelna G. y Moeller-Chávez G. E. (2012). La biofiltración sobre materiales orgánicos, nueva tecnología sustentable para tratar agua residual en pequeñas comunidades e industrias. Tecnol. Cienc. Agua. 3, 153-161.

Garzón-Zúñiga M.A., Hanssen I., Buelna G. y Bellemare G. (2006). Biofiltración sobre materiales orgánicos de regiones tropicales y subtropicales: Desarrollo IMTA - CRIQ (2 $2^{\mathrm{a}}$ Etapa). Informe de Proyecto Instituto Mexicano de Tecnologia del Agua IMTA- TC0606.1. Jiutepec, Morelos, México, 48 pp.

Garzón-Zúñiga, M.A., Lessard, P. Aubry G. y Buelna G. (2005). Nitrogen elimination mechanisims in an organic media aereated biofilter treating pig manure. Environ. Technol. 26, 361-371. DOI: 10.1080/095933332608618552.

Garzón-Zúñiga M.A., Sandoval-Villasana A.M y MoellerChávez, G. (2011). Biological removal of the AO24 azo dye and reduction of toxicity and genotoxicity. Water Environ. Res. 83, 107-115.
Garzón-Zúñiga M.A., Tomasini-Ortíz A.C., MoellerChavez G., Hornelas-Uribe Y., Buelna G. y MijaylovaNacheva P. (2008). Enhanced pathogen removal in on-site biofiltration systems over organic filtration materials. Wat. Practice and Technol. 3, 1-8. DOI: 10.2166/wpt.2008.053.

Gilbert D., Amblard C., Bourdier G., Francez A.J. y Mitchell E.A.D. (2000). Le régime alimentaire des Thécamoebiens (Protista, Sarcodinia). Année Biol. 39, 57-68. DOI: 10.1016/S0003-5017(00)80001-X.

He S.B., Yan L., Kong H.N., Liu Z.M., Wu D.Y., y Hu Z.B. (2007). Treatment efficiencies of constructed wetlands for eutrophic landscape river water. Pedosphere 17, 522-528. DOI: 10.1016/S1002-0160(07)60062-9.

Korkusuz E.A., Beklioglu M. y Demirer G.N. (2007). Use of blast furnace granulated slag as a substrate in vertical flow reed beds: field application. Bioresour. Technol. 98, 2089-2101. DOI: 10.1016/j. biortech.2006.08.027.

Lens P., Vochten P., Speleers L. y Verstraete W. (1994). Direct treatment of domestic wastewater by percolation over peat, bark and woodchips. Water Res. 28, 17-26. DOI: 10.1016/0043-1354(94)90115-5.

Metcalf y Eddy (2003) Wastewater engineering. Treatment and reuse. 4a ed. McGraw Hill, Nueva York, EUA, 1819 pp.

Mietto A., Politeo M., Breschigliario S. y Borin M. (2014). Temperature influence on nitrogen removal in a hybrid constructed wetland system in Northen Italy. Ecol. Eng. 75, 291-302. DOI: 10.1016/j.ecoleng.2014.11.027.

Molle P., Lienard A., Grasmick A., Iwema A., y Kabbabi A. (2005). Apatite as an interesting seed to remove phosphorus from wastewater in constructed wetlands. Water Sci. Technol. 51, 193-203.

Nicolau A., Martins M.J., Mota M. y Lime N. (2005). Effect of copper in the protistan community of activated sludge. Chemosphere 58, 605-614. doi:10.1016/j. chemosphere.2004.08.096.

Rivas A., Barceló-Quintal I. y Moeller G.E. (2011). Pollutants removal in a multi-stage municipal wastewater system comprised of constructed wetlands and a maturation pond in a temperate climate. Wat. Sci. Tech. 64, 980-987. DOI: 10.2166/wst.2011.731.

SEMARNAT (1996). Norma Oficial Mexicana NOM001-SEMARNAT-1996. Que establece los límites máximos permisibles de contaminantes en las descargas de aguas residuales en aguas y bienes nacionales. Secretaría de Medio Ambiente y Recursos Naturales. Diario Oficial de la Federación. 6 de enero de 1997.

SEMARNAT (2012). Norma Oficial Mexicana NMXAA-113-SCFI-2012. Que establece el método para la cuantificación e identificación de huevos de helminto en aguas residuales y residuales tratadas con el fin de 
evaluar la calidad del agua y la eficiencia de los sistemas de tratamiento de la misma. Secretaría de Medio Ambiente y Recursos Naturales. Diario Oficial de la Federación. 9 de abril de 2013.

Seo D., Cho J., Lee H. y Heo J.(2005). Phosphorus retention capacity of filter media for estimating the longevity of constructed wetland. Water Res. 39, 2445-2457. DOI: $10.1016 /$ j.watres.2005.04.032.

USEPA (2002). EPA-625-R-00-008. Onsite wastewater treatment systems, United States Environmental Protection Agency. Manual. Washington, D.C., EUA, $367 \mathrm{pp}$.

USEPA (2004). EPA-625-R-04-108. Guidelines for Water Reuse. United States Environmental Protection Agency. Manual. Washington, D.C., EUA, 480 pp.
Vigueras-Cortés J. M., Viallanueva-Fierro I., Garzón-Zúñiga M.A., Navar-Chaidez J.J., Hernández-Rodríguez C. y Chairez-Hernández I. (2013). Performance of biofilters packed with agave fiber in separation plates of municipal wastewater treatment. Wat. Sci. Tech. 68, 599-607. DOI: 10.2166/wst.2013.285.

Wu H., Zhang J., Hao H., Guo W., Hu Z., Liang S., Fan J. y Liu H. (2015). A review on the sustainability of constructed wetlands for wastewater treatment: Desing and operation. Bioresource Technol. 175, 594-601. DOI: 10.1016/j.biortech.2014.10.068.

Xu D.F., Xu J.M., Wu J.J., and Muhammad A. (2006). Studies on the phosphorus sorption capacity of substrates used in constructed wetland systems. Chemosphere 63, 344-352. DOI: 10.1016/j.chemosphere.2005.08.036. 